\title{
Nutritional control of gene expression in Drosophila larvae via TOR, Myc and a novel cis- regulatory element
}

\author{
Ling Li ${ }^{1}$, Bruce A Edgar ${ }^{1}$, Savraj S Grewal ${ }^{2,3^{*}}$
}

\begin{abstract}
Background: Nutrient availability is a key determinant of eukaryotic cell growth. In unicellular organisms many signaling and transcriptional networks link nutrient availability to the expression of metabolic genes required for growth. However, less is known about the corresponding mechanisms that operate in metazoans. We used gene expression profiling to explore this issue in developing Drosophila larvae.

Results: We found that starvation for dietary amino acids (AA's) leads to dynamic changes in transcript levels of many metabolic genes. The conserved insulin/PI3K and TOR signaling pathways mediate nutrition-dependent growth in Drosophila and other animals. We found that many AA starvation-responsive transcripts were also altered in TOR mutants. In contrast, although PI3K overexpression induced robust changes in the expression of many metabolic genes, these changes showed limited overlap with the AA starvation expression profile. We did however identify a strong overlap between genes regulated by the transcription factor, Myc, and AA starvation-responsive genes, particularly those involved in ribosome biogenesis, protein synthesis and mitochondrial function. The consensus Myc DNA binding site is enriched in promoters of these AA starvation genes, and we found that Myc overexpression could bypass dietary AA to induce expression of these genes. We also identified another sequence motif (Motif 1) enriched in the promoters of AA starvation-responsive genes. We showed that Motif 1 was both necessary and sufficient to mediate transcriptional responses to dietary AA in larvae.

Conclusions: Our data suggest that many of the transcriptional effects of amino acids are mediated via signaling through the TOR pathway in Drosophila larvae. We also find that these transcriptional effects are mediated through at least two mechanisms: via the transcription factor Myc, and via the Motif 1 cis-regulatory element. These studies begin to elucidate a nutrient-responsive signaling network that controls metabolic gene transcription in Drosophila.
\end{abstract}

\section{Background}

The availability of extracellular nutrients is a key determinant of eukaryotic cell growth. In single cell organisms, such as budding yeast, an extensive signal transduction and transcriptional network links extracellular nutrients to the expression of metabolic genes $[1,2]$. These networks are essential for the proper control of cell growth and proliferation [3,4]. In metazoans, nutrition controls both cell and organismal growth. These effects require a complex interplay between cellautonomous and systemic responses to nutrient

\footnotetext{
* Correspondence: grewalss@ucalgary.ca

${ }^{2}$ Clark H. Smith Brain Tumour Center, Southern Alberta Cancer Research Institute, Calgary, Alberta, T2N 4N1, Canada
}

availability. However, the cellular mechanisms that mediate these effects are still poorly understood.

In Drosophila dietary amino acids are essential for larval growth and development [5]. Amino acid starvation leads to inhibition of cell growth and cell cycle progression in virtually all larval tissues. The Drosophila insulin/PI3 kinase pathway plays a central role in nutritionregulated growth [6]. In response to dietary protein, Drosophila insulin-like peptides (Dilps) are released from neurosecretory cells. These Dilps act in an endocrine manner and trigger growth by binding to the insulin receptor and activating a conserved PI3 kinase (PI3K) and Akt kinase signaling pathway in all larval tissues [7]. The TORC1 (TOR complex 1) pathway is a second important mediator of nutritional inputs. The 
TORC1 protein complex contains the TOR kinase and can be activated in a cell autonomous manner in response to extracellular nutrients and amino acids, and also as a downstream target of the insulin/PI3K pathway $[8,9]$. As such, insulin/PI3K and TOR can function either independently or as a linear signaling cascade, depending on the stimulatory inputs. Importantly, cell autonomous activation of both insulin/PI3K and TOR can bypass the requirement for dietary protein to control growth in Drosophila larval tissues [6,10,11].

Much is known about the complex signaling inputs and crosstalk between the insulin/PI3K and TOR pathways. These regulatory inputs are highly conserved and control cell and organismal growth in all animals. Many oncogenes and tumor suppressors are part of these signaling inputs, and aberrant PI3K and TOR signaling is a feature of many different cancers [12]. However, less is known about the growth regulatory outputs of these pathways [13]. Given the important role of transcription in mediating effects of nutrients in budding yeast it is likely that changes in gene expression are important in metazoans too. Recent studies in Drosophila support this notion [13-17]. For example, the FOXO transcription factor, a conserved target of the insulin/PI3K pathway, can regulate gene expression in response to starvation in larvae, in part through secondary regulation of the transcription factor Myc [16]. Also, nutrientdependent TOR signaling controls ribosomal RNA (rRNA) transcription in larvae, via the conserved Pol I factor, TIF-IA [18]. Finally, in Drosophila adults, brain specific insulin/PI3K signaling can control gene expression through the transducer of regulated CREB (TORC) transcriptional regulator [19].

In this paper we report gene expression profiling of both AA starvation, and the PI3K and TOR pathways in Drosophila larvae. We find that starvation for dietary AA induces robust and dynamic changes in metabolic gene expression. By comparing gene expression profiles we suggest that some, but not all, transcriptional effects of starvation are mediated through the TOR pathway. Interestingly, although overexpression of PI3K induces robust changes in metabolic gene expression, these changes show a limited overlap with the AA starvation expression profile. Instead, we find that many of the transcriptional responses to changes in dietary protein are mediated via the transcription factor dMyc. Finally, we identified DNA motifs in promoters of proteinresponsive genes and show that a previously uncharacterized motif (Motif 1) confers AA starvation-responsiveness in transgenic reporter lines. Together these studies begin to elucidate a nutrient-responsive signaling network that controls metabolic gene transcription in Drosophila.

\section{Results}

Gene expression profiling in protein-starved larvae

Starvation for dietary amino acids (AAs) leads to growth arrest and cell cycle inhibition in virtually all polyploid cells of the larval organs [5]. We were therefore interested in identifying AA starvation-induced transcriptional changes occurring in the bulk of these tissues. Thus we used cDNA microarrays to analyze gene expression profiles in whole larvae. We starved second instar larvae by floating them in $20 \%$ sucrose/PBS at 62 $\mathrm{h}+/-3 \mathrm{hrs}$ after egg deposition, and then examined gene expression profiles at various time points following AA starvation. For all time points, comparisons were made to zero hour time point control larvae.

Of the six thousand genes from DGC-1 on the microarray chips, Significance Analysis of Microarrays (SAM) analysis indicated that a total of 1888 were altered by AA starvation (Table 1 and Additional File 1). We observed both increases and decreases in gene expression. Significant changes were seen at four-hour starvation suggesting a very rapid transcriptional response to AA starvation. Strong effects were seen at 4 days post starvation with decreases in 636 transcripts and increases in 647 transcripts, which together make up almost a quarter of all genes analyzed on the microarrays (Figure 1 and Table 1 ). Importantly, most AA-starvation changes in transcript levels were reversed upon re-feeding (Figure 1A). For example, half (172 of 325, $53 \%)$ the genes that were either suppressed or enhanced at $24 \mathrm{~h}$ of AA starvation were reversed within $4 \mathrm{~h}$ of refeeding larvae with a protein rich diet. Similarly, oneday of re-feeding reversed many (254 of 636, 40\%) changes in gene expression caused by 4-day starvation. Cluster analysis identified several groups of genes with different expression kinetics. These clusters suggest a dynamic temporal pattern of gene expression in response to AA starvation and re-feeding (Additional File 2).

Based on the types of genes affected, our data pointed to broad transcription-dependent changes in cellular and organismal metabolism upon AA starvation. For example, at $24 \mathrm{~h}$ starvation 325 transcripts were reduced. Of these 156 were of unknown function (i.e. no Gene Ontology (GO) annotation). However, of the transcripts with GO term annotations, approximately one-third were involved in ribosome biogenesis and protein synthesis while another one-third of reduced transcripts were annotated as being involved in nutrient metabolism, including many genes required for mitochondrial function (Figure B and Additional File 3). Many genes involved in proteolysis were also altered in response to amino acid withdrawal (Additional File 4). In particular, several autophagy related genes (ATGs) were induced, 


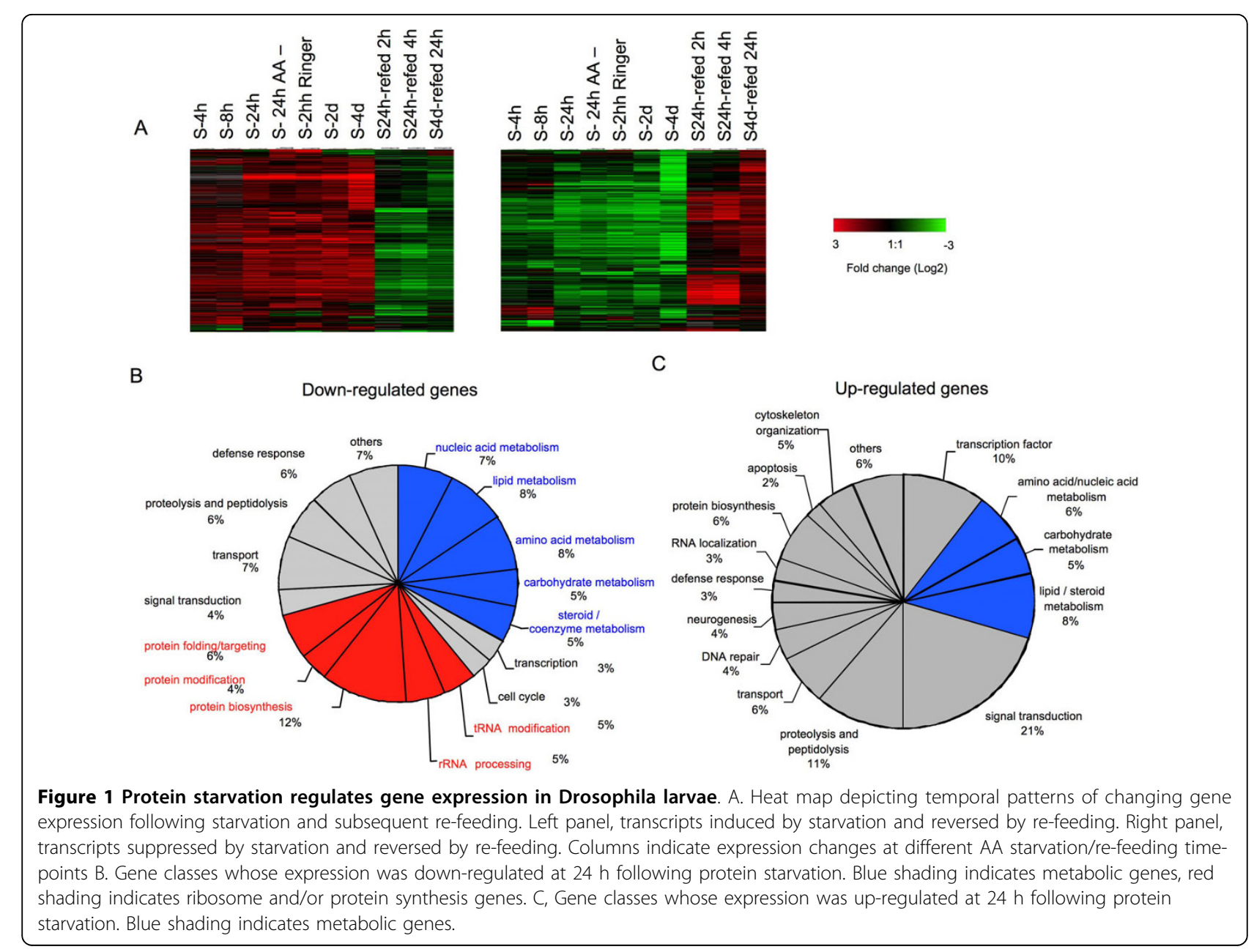

in some cases within 4-8 $\mathrm{h}$ of protein starvation (Additional File 4). Expression of genes coding for regulators of lipid and steroid metabolism was also affected by starvation (Additional File 5). Interestingly although AA starvation leads to a block in cell cycle progression in most larval tissues, particularly the endoreplicating tissues which make up the bulk of the animal, transcripts coding for cell cycle genes were largely unaffected by starvation (Additional File 6). Together these data suggest a dynamic, rapid transcriptional response to protein starvation that both increases and decreases gene expression and alters larval metabolism.

We also compared the gene expression profiles obtained from three different $24 \mathrm{hr}$ starvation conditions (complete starvation, sucrose-only diet, standard diet minus protein) (Additional File 7). In general there was

Table 1 Summary of AA starvation-regulated genes.

\begin{tabular}{lcccc}
\hline & Down-regulated genes & Up-regulated genes & Total & \% of total genes on the microarray \\
\hline AA starved 4 h & 210 & 313 & 523 & 9.4 \\
AA starved 8 h & 203 & 226 & 429 & 7.7 \\
AA starved 24 h & 325 & 260 & 585 & 10.6 \\
Complete food minus AA 24 h & 256 & 267 & 523 & 9.4 \\
Complete starve 24 h & 350 & 358 & 708 & 12.8 \\
AA starved 2 d & 434 & 393 & 827 & 14.9 \\
AA starved 4 d & 636 & 647 & 1283 & 23.2 \\
AA Starved 24 h Refed 2 h & 138 & 274 & 412 & 7.4 \\
AA Starved 24 h Refed 4 h & 202 & 378 & 580 & 10.4 \\
AA Starved 4 d Refed 1 d & 115 & 313 & 428 & 7.7 \\
\hline
\end{tabular}


a strong overlap between affected genes across each condition, particularly with respect to genes whose expression was down-regulated. For example, $65 \%$ of transcripts whose expression was reduced upon complete starvation were also suppressed in the sucroseonly condition. These data suggest that a key, limiting factor for gene expression is availability of dietary AA (Figure 1A and Additional File 7). Nevertheless, expression of many genes was affected in only one of the starvation conditions, possibly reflecting an additional role for dietary sugars and fats in the control of gene expression. This was particularly true for transcripts whose expression was increased upon starvation (Additional File 7).

Transcriptional changes induced by PI3K and TOR signaling pathways

We next examined the gene expression profiles associated with the TOR and insulin/PI3K signaling pathways. A null allele of TOR phenocopies AA-starvation and leads to growth arrest in early larval development ( $72 \mathrm{~h}$ after egg deposition (AED) [20]. Expression profiling revealed that a total of 464 transcripts were significantly altered in $t o r^{-1-}$ mutant larvae at $72 \mathrm{~h}$ AED (Figure 2A, B). As with AA starvation, these transcripts coded for genes largely involved in metabolism (Figure 2A, B and Additional File 1), although considerably fewer transcripts were altered in tor $^{-1-}$ mutants. Nevertheless, $61 \%$ of transcripts that were significantly reduced in $\mathrm{tor}^{-1-}$ mutants were also significantly reduced in protein-starved larvae (Figure 2C). These altered transcripts comprised approximately $19 \%$ of starvation-suppressed transcripts. In addition $46 \%$ of transcripts increased in tor $^{-1-}$ mutants were also increased in starved larvae. This suggests that inhibition of TOR partially mediates the transcriptional response to AA starvation.

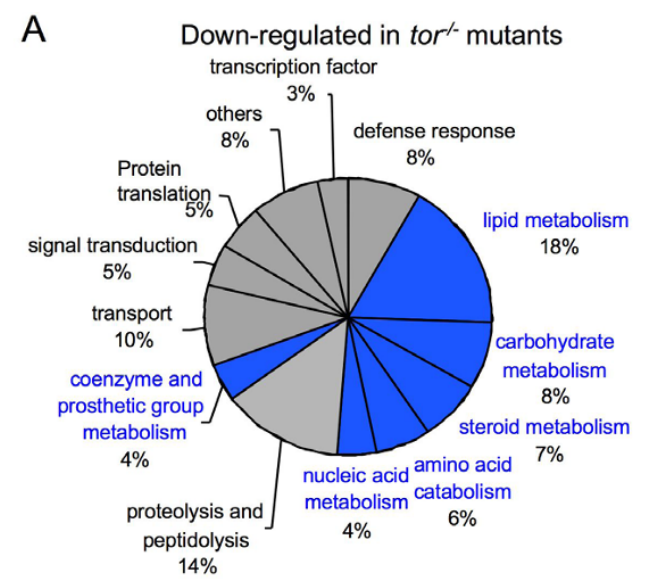

C

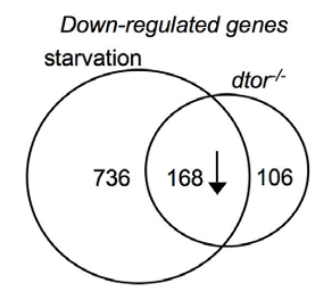

Up-regulated genes

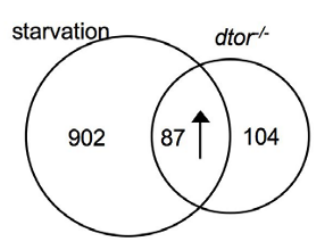

B

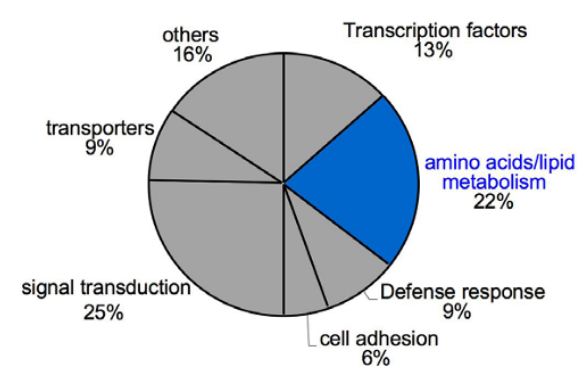

D

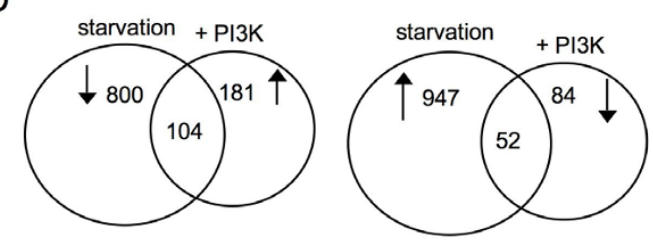

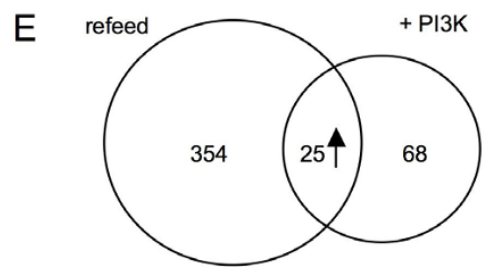

Figure 2 Expression profiling of TOR mutants and PI3 kinase overexpression. A. Gene classes whose expression was down-regulated in tor $^{-1-}$ mutants. Blue shading indicates metabolic genes. B, Gene classes whose expression was upregulated in tor $r^{-1-}$ mutants. Blue shading indicates metabolic genes. C, Venn diagram showing the overlap in genes regulated by protein starvation and TOR mutants. Left panel, genes down-regulated in both conditions. Right panel, genes up-regulated in both conditions. D, Venn diagram showing the overlap in genes whose expression is reciprocally regulated by protein starvation and PI3 kinase overexpression. E. Venn diagram showing the overlap in genes regulated in AA-starved animals by either re-feeding with complete food or by PI3 kinase overexpression. 
The Insulin/PI3K pathway is activated in response to nutrient availability, and is thought to be an important regulatory input to the TOR pathway. We over expressed a PI3K transgene in third instar larvae and examined gene expression profiles at 14 and $24 \mathrm{~h}$ postinduction. At the early time, expression levels of 118 transcripts were altered ( 87 increased and 39 decreased), whereas at $24 \mathrm{~h}$ this increased to 421 transcripts (285 increased and 341 decreased). The bulk of the altered transcripts coded for genes required for carbohydrate and lipid metabolism (Additional File 1 and Additional File 8). Other affected genes were involved in protein biosynthesis, transcription and mitochondrial function (Additional File 1 and Additional File 8).

Given the role of PI3K in nutrition-dependent signaling, we predicted a reciprocal relationship between genes affected by PI3K overexpression and starvation. However, of the 285 genes increased at $14 \mathrm{~h}$ post PI3K-induction in fed animals, only 48 (17\%) were decreased in $24 \mathrm{~h}$ AA-starved larvae (48\% of all $24 \mathrm{~h}$ starvation suppressed transcripts) (Figure 2D). We also compared gene expression profiles of $24 \mathrm{~h}$ AA starved larvae that were either refed or in which PI3K was overexpressed for $14 \mathrm{hrs}$. As described previously, $4 \mathrm{hr}$ of re-feeding reversed $52 \%$ of the $24 \mathrm{~h}$ starvationmediated changes in gene expression. However, we found only a small overlap (17\%) in the re-feeding expression profiles versus the expression profile associated with overexpressing PI3K in starved animals (Figure 2E). These data suggest that only a small fraction of AA starvation-regulated genes respond to overactivation of the insulin/PI3K pathway.

\section{Myc regulates the expression of starvation responsive genes}

We previously showed that overexpression of the transcription factor Myc in larvae could increase the expression of many metabolic genes, particularly those involved in ribosomal biogenesis and protein synthesis genes [21]. These Myc regulated genes strongly overlapped with many AA starvation-responsive genes identified in this report. For example, $60 \%$ of genes induced by AA-starvation and re-feeding are also induced by 14 hr Myc overexpression in larvae. This overlap in regulation was particularly true for genes involved in ribosome biogenesis and protein synthesis (Figure 4). A MEME motif search identified the consensus Myc binding site (E-box - CACGTG) as being highly enriched in promoters of Pol I and Pol III genes and rRNA processing genes, and genes required for mRNA translation. Interestingly, although the ribosomal protein genes were all regulated by Myc, very few had canonical Myc E-boxes in their promoter. This suggests Myc may function via intermediate transcription factors to control the expression of ribosome protein genes.
We also compared the gene expression profiles of Myc homozygous null mutant and AA-starved animals. Myc null mutants are lethal and arrest their growth early in larval development, and show changes in gene expression that are largely the reciprocal of those mediated by Myc overexpression [22]. We identified a strong overlap between genes whose transcripts levels were reduced in both AA-starved animals and $24 \mathrm{~h} \mathrm{myc}^{-1-}$ homozygous mutant larvae (51\% of Myc-regulated genes) (Figure 3B). Of the genes that overlap, nearly all contain E-boxes (including half with consensus Myc binding sites -CACGTG). This overlap suggests that Myc may mediate transcriptional activation induced by AA-dependent signaling. In contrast less then $10 \%$ of genes whose expression was increased upon AA-starvation were altered in $m y c^{-1-}$ mutants.

To further examine the role of Myc in AA-dependent transcription we analyzed the gene expression profiles in $24 \mathrm{~h}$ AA starved larvae in which we over expressed a Myc transgene for a further $24 \mathrm{~h}$. We found that many of the changes in gene expression induced by starvation could be reversed by overexpression of Myc (Figure 3C). In particular, we found that $70 \%$ of transcripts increased by Myc overexpression in AA-starved larvae were also increased upon re-feeding in starved animals (these genes accounted for $56 \%$ of all refeeding-induced transcripts - Figure 3D). Together, these data suggest that Myc overexpression can bypass starvation to control the expression of many AA-responsive genes. This contrasts with our findings with PI3K overexpression. Not surprisingly, we found very little overlap (9\% of Mycinduced genes and $13 \%$ of Myc-repressed genes) between the gene expression profiles of Myc-versus PI3K-overexpression in third instar larvae (Figure 4)

\section{Other potential nutrient-dependent transcriptional regulators}

A goal of these studies was to identify potential cis-regulatory DNA motifs and corresponding transcription factors that control transcription of nutrient-regulated genes. As described above Myc is a strong candidate and many AA starvation-regulated genes contained canonical Myc E-boxes within their promoters. We used the MEME motif search tool to try and identify other motifs enriched within the promoters of AA starvationresponsive genes. We analyzed two clusters of genes whose expression profiles showed strong regulation by AA starvation (Figure 5A). Cluster A contained genes whose transcript levels were rapidly induced and maintained by AA starvation, and then rapidly decreased by AA refeeding (Fig 5A). Cluster B contained genes whose transcripts were regulated in the opposite manner. Two of the top motifs identified in this bioinformatic search were an E-box bHLH transcription factor binding site (found in 14/42 Cluster A genes and 27/46 of Cluster B 


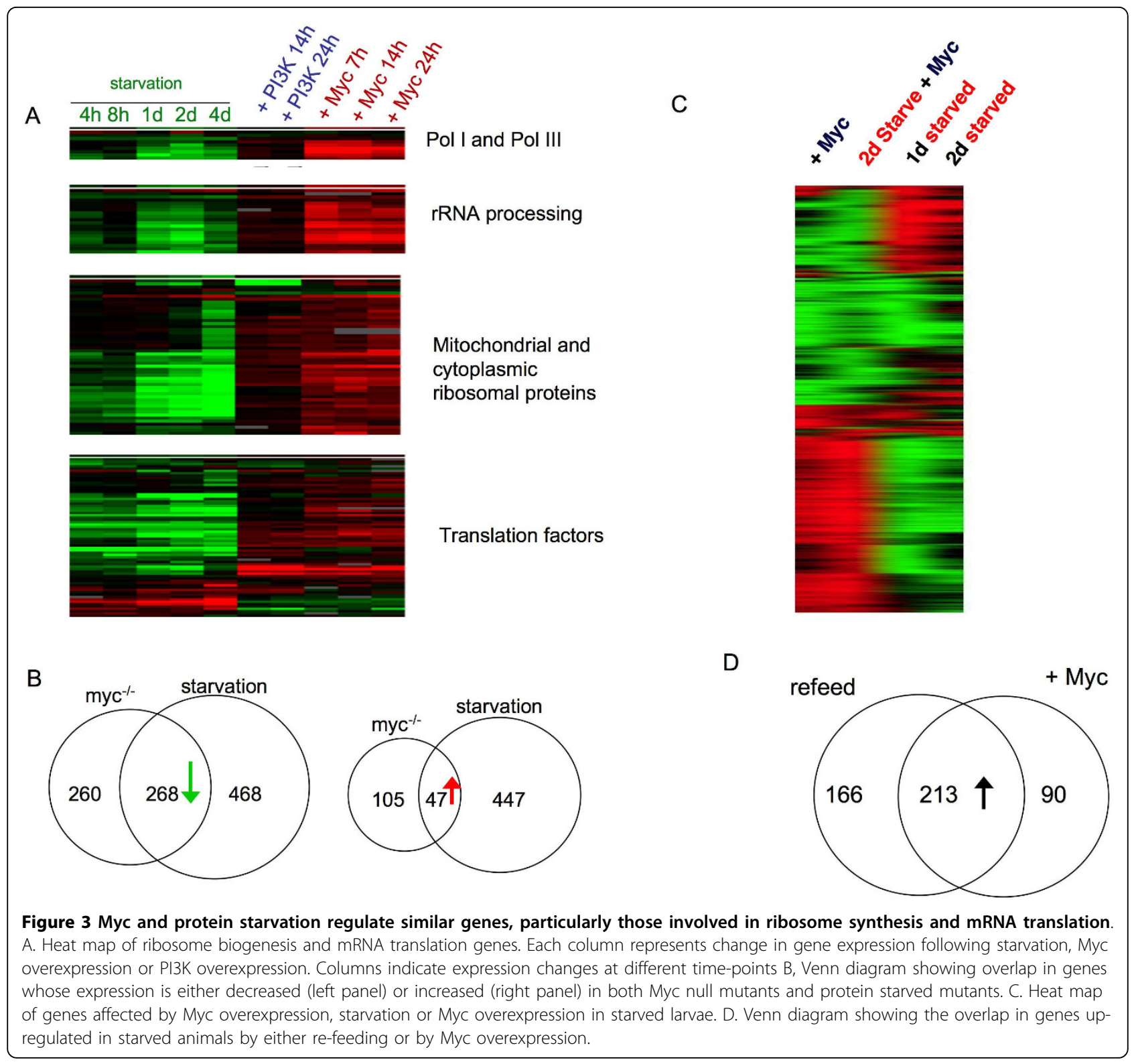

genes, $\left.\mathrm{p}<5 \times 10^{-4}\right)$, and a previously identified motif, Motif 1, (23/42 Cluster A genes and 21/46 of Cluster B genes $\mathrm{p}<6.4 \times 10^{-6}$ ) (Figure $5 \mathrm{~B}$ ). We found that $9 / 46$ cluster B genes and 10/46 cluster A genes contained both motifs. We also performed a similar MEME analysis on four non-overlapping groups of 40 genes whose expression was not altered by AA starvation (data not shown). In each case MEME analysis did not identify either Motif1 or an E-box in the promoters of these genes, suggesting that both motifs may be enriched in promoters of starvation-regulated genes. Motif 1 was previously identified as a core promoter element of many Drosophila genes [23] and we found it was close to the transcription start site of both Cluster A and B genes. In order to test the importance of Motif 1, we generated promoter-GFP reporter lines from seven genes from both cluster A and B (cluster A - CG7686, CG1135, CG6311, CG32486; cluster B - CG8470, CG6459, CG9539). In each case GFP was expressed in all tissues examined and mutation of Motif1 in each reporter abolished visible GFP expression (data not shown). We next used qRT-PCR to examine expression of GFP reporter for a gene from each of the two clusters: CG8470 from cluster B, and CG6311 from cluster A. Upon AA starvation, CG8470-GFP mRNA expression was reduced, while expression from CG6311-GFP was induced (Figure 5C, D). These changes are similar to alterations in endogenous transcript levels as measured 


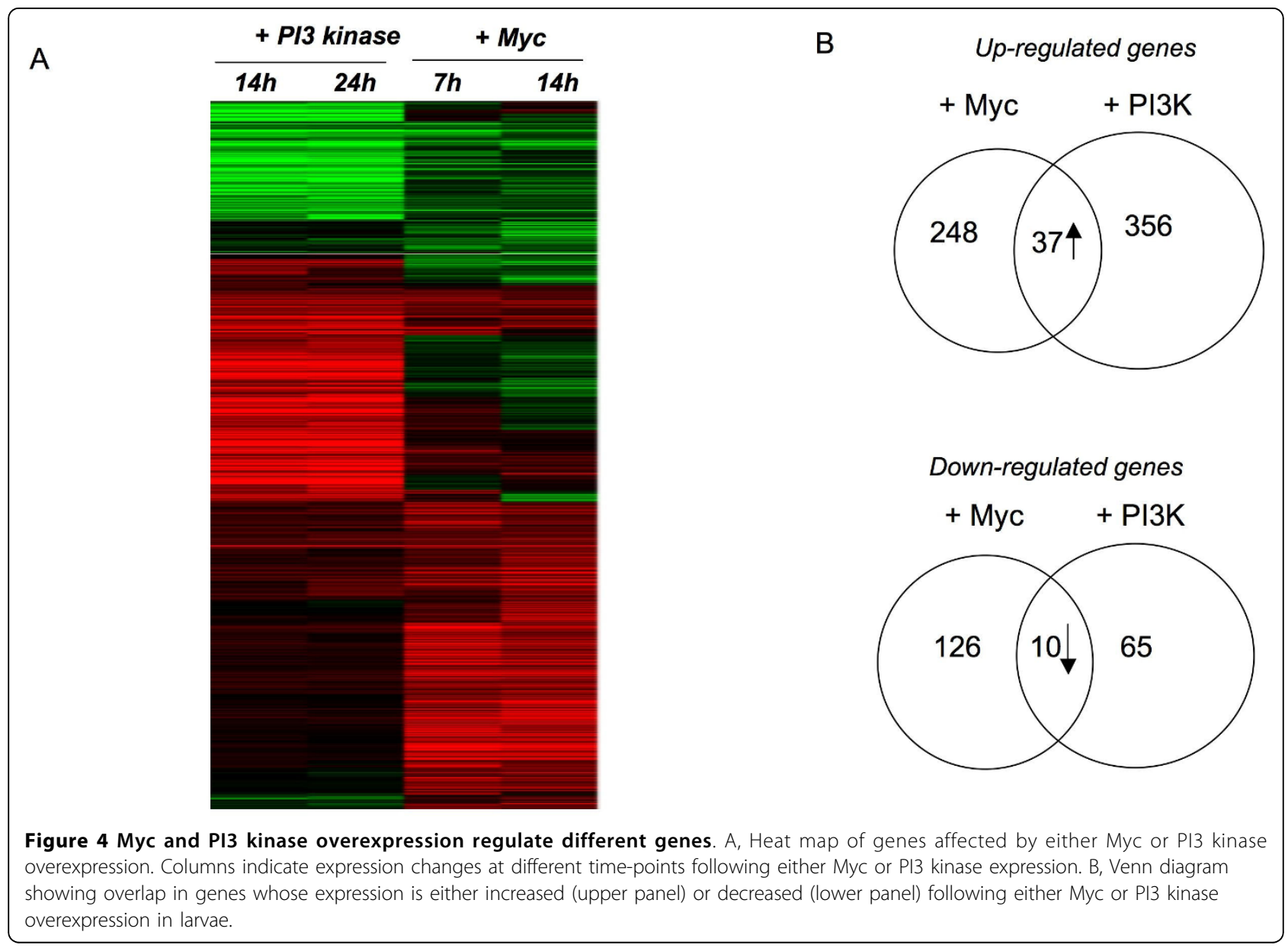

by Northern blot (data not shown) and microarray hybridization, consistent with the notion that the AA starvation-induced changes in mRNA levels are due to alterations in transcription. When we mutated Motif 1 in both reporters, expression was markedly reduced (Figure 5C, D). More importantly, we also found that the weak GFP expression from these Motif 1 mutated reporters was no longer starvation-responsive. We next generated several transgenic lines containing reiterated Motif 1 with a basal hsp promoter. This artificial Motif1 promoter was sufficient to drive GFP expression in all larval tissues examined (wing discs, gut, fat body, salivary gland). Interestingly, we found that GFP mRNA expression from this reporter was reduced when larvae were AA-starved (Figure 5E). These data suggest Motif 1 may bind a factor(s) required for transcription of AAstarvation responsive genes and whose activity may be regulated by AA availability. We used GOMO (Gene Ontology for Motifs) analysis to identify GO-terms that are significantly associated with genes containing motif 1 within their upstream regions (Additional File 9). The top scoring hits in this analysis were genes that were broadly involved in cell metabolism and cell division, based on GO definitions of molecular function and biological process. Interestingly, GOMO analysis also identified genes involved in ribosome synthesis based on the GO term of cellular component (Additional File 9). 3). We examined this further using MEME analysis of the upstream regions of ribosome biogenesis gene. Of the cluster of 82 ribosome synthesis genes that we examined, we identified both motif 1 (44 genes, $\mathrm{p}<1 \times 10^{-}$ ${ }^{27}$ ) and the consensus Myc E-box (42 genes, $\mathrm{p}<5 \times 10^{-}$ $\left.{ }^{9}\right)$ as two of the top motifs, with 13 genes containing both motifs. Together these analyses suggest that transcription through an E-box and/or motif1 links AAdependent signaling to metabolic gene expression, particularly genes involved in ribosome biogenesis and protein synthesis.

\section{Discussion}

Nutritional control of gene expression in Drosophila larvae

Starvation for dietary AA leads to profound changes in metabolism of Drosophila larvae. These changes 
include decreases in ribosome and protein synthesis $[18,24,25]$, alterations in the storage and metabolism of fat and carbohydrate $[6,25,26]$, and induction of autophagy [27]. These alterations are essential for arresting larval growth and development, and maintaining homeostasis under poor nutrient conditions[24,25,28]. Our microarray data suggest that many of these changes are mediated through nutrient-dependent modulation of gene expression. For example, mRNA levels of both protein synthesis and ribosome biogenesis genes are reduced upon AA starvation. Similarly genes involved in mitochondrial biogenesis and function, such as mitochondrial ribosomal proteins and a mitochondrial transcription factor, TFAM, are also suppressed by AA starvation. In contrast, several genes involved in proteolysis and autophagy are rapidly induced by removal of dietary AA.
A previous report also described both protein and complete nutrient starvation responses in whole larvae [14]. Although this study used different protocols to analyze gene expression, similar, but fewer, genes were identified compared to our study. More recently, Telemann et al (2008) [16] examined transcriptional responses to complete starvation in either fat or muscle. They identified many genes whose regulation was specific to either tissue, but they also reported changes in similar classes of genes especially those involved in ribosome synthesis, mRNA translation and mitochondrial biogenesis. Our analyses were done using whole larvae therefore we are probably identifying strong transcriptional changes that are occurring in most larval cells. Together, these previous reports and our data identify the major changes in metabolic gene expression mediated by dietary AA in Drosophila larvae.

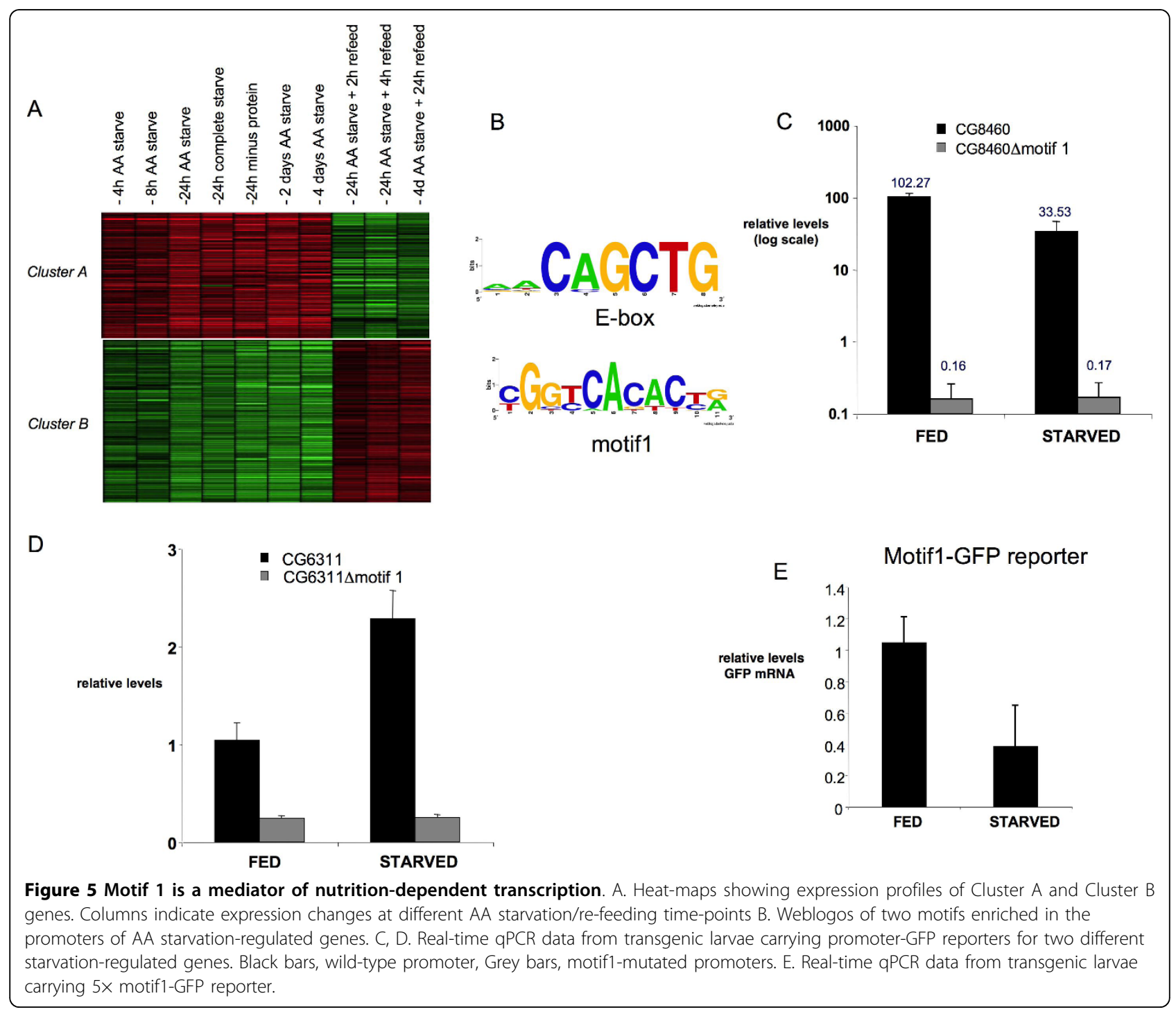


The bulk of the larval organs (such as the gut, fat body and muscle) are composed of polyploid cells which all undergo nutrition-dependent endoreplication $[5,29]$. AA starvation leads to a shutdown of cell cycle progression in these tissues [5,29]. However, our microarray analyses on starved larvae did not reveal significant changes in mRNA levels of cell cycle regulators. This finding suggests that nutrition-dependent control of cell cycle progression may occur through post-transcriptional changes in cell cycle genes. This type of regulation has been described in yeast in which nutrientdependent alterations in ribosome synthesis and mRNA translation can control translation of cell cycle genes [30].

\section{Transcript profiling of the PI3K and TOR signaling pathways}

The insulin/PI3K and TOR signaling pathways are the major mediators of nutrition-dependent cell and organismal growth in Drosophila larvae. Our microarray analyses allowed us to compare the expression profiles of AA starvation with modulation of PI3K and TOR signaling. These comparisons suggest that only about half of genes whose expression is altered by AA starvation also respond to modulation of PI3K and/or TOR signaling. The strongest overlap was between TOR and AA starvation-regulated genes. Virtually all of these overlapping genes were involved in the control of some aspect of cellular metabolism, particularly the control of carbohydrate and lipid metabolism. However, we did see some notable differences between starvation and TOR mutants. In particular, TOR mutants showed little change in the expression of ribosome biogenesis and protein synthesis genes. TOR mutants have reduced nucleolar size and lower levels of rRNA synthesis $[18,20]$, two effects that are indicative of reduced ribosome synthesis and that are phencopied by protein starvation [18]. Also, inhibition of TOR in cultured Drosophila cells does inhibit expression of ribosome biogenesis genes [31]. It is possible these differences may reflect tissue specific effects (i.e. cell culture versus larvae). However, it is likely that many of the effects of TOR on ribosome synthesis may be mediated through post-transcriptional control of downstream effectors e.g. TIF-IA [18] and 4E-BP. Indeed many of the nutritiondependent functions of TOR probably reflect transcription-independent effects of TOR on processes such as mRNA translation [32], autophagy [28] and endocytosis [33].

We saw a much weaker overlap between genes affected by AA starvation versus PI3K overexpression. For example, given the role of insulin/PI3K signaling in nutrition-dependent growth, we predicted that genes suppressed by starvation might be induced by PI3K overexpression. However, this prediction was correct for only a small subset (12\%) of AA-starvation suppressed genes. In particular we found that genes involved in ribosome biogenesis and protein synthesis, two processes strongly influenced by protein starvation, were generally unaffected by PI3K. Thus, not all AA starvation-regulated genes respond to activation of the insulin/PI3K pathway. The transcription factor FOXO is a conserved downstream target of the PI3K pathway. High PI3K activity leads to nuclear export and inhibition of FOXO [34]. It has been suggested that many transcriptional responses to $\mathrm{PI} 3 \mathrm{~K}$ activation are mediated through repression of FOXO activity. Indeed, a recent paper implicated FOXO in mediating many transcriptional effects of AA starvation [16]. This suggestion would seem at odds with our comparison of AA starvation and PI3K-responsive genes. These differences between our findings with PI3K overexpression and previous examination of FOXO mutants may be due to different experimental approaches. For example, we examined gene expression following overexpression of PI3K in both fed and starved larvae, whereas Telemann et al. examined starvation expression profiles in either fat or muscle of FOXO mutants. In addition, it is likely that many of the effects of PI3K overexpression on both transcription and growth can occur independently of FOXO. For example, FOXO mutants have no growth phenotypes in nutrient rich food [35], while overexpression of PI3K can induce marked cell growth under identical conditions [6], suggesting that the effects of PI3K involve more than FOXO inhibition alone.

\section{Myc as a downstream effector of nutrition-dependent transcription}

One key finding from our work was the role of Myc in nutrition-mediated gene expression. We found that the gene expression profiles of both Myc mutants and Mycoverexpression exhibit a strong overlap with AA starvation-responsive genes. Many of the overlapping genes were involved in ribosome synthesis, mRNA translation and mitochondrial function. Moreover, we showed that Myc overexpression could bypass a requirement for AA and reverse repression of many of genes induced by starvation. These findings suggest that Myc acts as a downstream mediator of AA-dependent regulation of gene transcription and expression of metabolic genes in Drosophila larvae. Most of these Myc- and AA starvation-sensitive genes contain either the consensus Myc binding site or alternate E-box motifs that may also bind Myc, within their promoters. A recent paper reported a similar enrichment of Myc binding sites within the promoters of genes inhibited by rapamycin treatment in cultured Drosophila S2 cells, particularly ribosome synthesis genes [16]. This latter study reported that Myc association with the promoters of these genes was inhibited by treatment with the TOR inhibitor, 
rapamycin [16]. Our in vivo 'by-pass' experiments extend these cell culture findings to show that Myc functions downstream of nutrition to control metabolic gene expression in developing larvae. Two recent reports indicated that Myc mRNA levels were FOXOregulated in a tissue specific manner [16,36], hence providing one potential mechanism by which Myc might be regulated downstream of AA availability. Regulation of Myc protein levels by either translation control or ubiquitin mediated-degradation may also be a downstream effect of AA.

An important finding from our work was the marked differences in the expression profiles of PI3K-overexpression versus Myc-overexpression. For example, we found that Myc induced expression of a large number ribosome biogenesis and protein synthesis genes (Fig 4) whereas PI3K-overexpression did not. Consistent with this, we previously reported that Myc overexpression had strong effects on rRNA synthesis, whereas PI3K overexpression had little or no effect [21]. Thus, while both Myc and PI3K can induce quantitatively similar increases in growth, comparisons between their gene expression profiles suggest qualitatively different effects of Myc versus PI3K on transcription and metabolism. Indeed, Myc and PI3K produce morphologically different effects on cell growth in the larval fat body [37]. Importantly, these differences between PI3K and Myc suggest that the effects of insulin/PI3K on transcription are probably in large part independent of Myc function. In contrast, a recent paper reported that in cultured Drosophila S2 cells, insulin treatment (and presumably increased PI3K activity) promoted Myc binding to the promoters of ribosome biogenesis genes and increased expression of these genes [16]. These results contrast with our data and may reflect differences between responses to increased PI3K activity in in vitro cultured S2 cells versus developing larvae. Nevertheless, our data do point to differences in the way PI3K and Myc regulate gene expression and cell growth in vivo. These qualitative differences between how PI3K and Myc function may have implications for situations in which growth is deregulated, such as cancer. Thus, increases in PI3K pathway activity and Myc may each induce distinct effects on metabolism, and consequently cooperate to drive aberrant cell growth and induce transformation [38]. In fact, increased Myc levels and overactivation of effectors of the PI3K pathway are observed in many cancers and may function synergistically to promote tumor progression [39].

\section{Motif1 as a AA starvation-regulated promoter element}

Nutrient dependent changes in gene transcription have been well studied in budding yeast [1,2]. In this unicellular organism alterations in extracellular levels of nutrients and amino acids can elicit widespread changes in transcription of large classes of metabolic genes, particularly those involved in ribosome biogenesis and protein synthesis $[2,4]$. In many cases the cis-regulatory promoter elements and corresponding DNA-binding factors that mediate these responses have been defined [2]. However, these factors often have no obvious homologs in metazoans. In Drosophila, our work and that of others suggests Myc and TORC2-CREB function as nutrition/PI3K/TOR-regulated transcription factors $[16,19]$. However, no additional factors have been described. In this paper we surveyed the promoters of genes whose expression is AA starvation-sensitive for enriched DNA motifs. One motif in particular (Motif1) emerged from this analysis. Motif 1 was previously identified as a core promoter element in a large number of genes in Drosophila, although the exact binding factor(s) are not known [23]. Our data suggest that Motif1 is required for transcription of AA starvation-responsive genes. Moreover, Motif-dependent transcription is inhibited by AA starvation. These findings raise the possibility that motif 1 is a binding site for AA starvationregulated transcription factor(s). It is likely that this putative factor(s) is an activator rather than a repressor since mutation of motif1 in the transgenic reporters led to reduced transcription as opposed to de-repression (and increased transcription). Given the location of motif 1 within the core promoters of many Drosophila genes and its proximity to the transcription start site [23], the factor(s) that bind Motif 1 may also either be, or associate with, core promoter factors. This view is consistent with emerging data suggesting that regulation of core promoter factors can control differential gene expression. For example, in mammalian cells, induction of myogenesis involves differential expression and recruitment of core transcription factors [40]. Also, in yeast the expression of stress- versus growth-regulatory genes in response to environmental cues depends on differential recruitment of core promoter factors [1]. We found Motif1 in the promoters of many classes of AA starvation-responsive metabolic genes, particularly those involved in protein synthesis and ribosome biogenesis. Moreover, a previous genome-wide binding analysis of $\mathrm{dMyc}$ showed that the presence of Motif1 significantly correlates with the presence of consensus Myc-binding sites [41]. These findings together with our data suggest that a putative motif-1-binding factor and Myc may function to control metabolic gene transcription in response to nutrient availability in Drosophila. Whether transcription through both promoter elements is cooperative or additive is unclear. However, since many AA starvation-responsive genes (e.g. cluster $\mathrm{A}$ and $\mathrm{B}$ genes, ribosome biogenesis genes) contain only one or other of the two motifs, motif1 and the E-box can probably function independently of each other. 


\section{Conclusions}

Our data show that AA starvation leads to widespread changes in metabolic gene expression in Drosophila larvae. These effects are mediated in large part through the TOR signaling pathway. We also identify at least two transcriptional mechanisms by which these changes occur: via the transcription factor, Myc, and through the Motif-1 cis-regulatory element.

\section{Methods}

\section{Fly strains and protocols}

The following fly stocks were used: ywhsflp122; +; +, ywhsflp122; +; UAS-dMyc, ywhsflp122; UAS-dp110, Act $>$ CD2>GAL4, UAS-GFP, w1118; $\mathrm{dTOR}^{\triangle \mathrm{P}} / \mathrm{CyO}-$ GFP.

All flies were raised at $25 \mathrm{C}$. For all starvation experiments wild-type embryos were collected on agar/grape juice plates. Fifty newly hatched larvae were transferred to vial of normal fly food supplemented with yeast paste. They were raised for $36 \mathrm{hr}(+/-3 \mathrm{hrs})$ and then starved by first washing with $1 \times$ PBS and then floating in $20 \%$ sucrose/PBS. Control (zero-hour) larvae were washed and then collected for subsequent RNA extraction. For longer starvation, the sucrose media was changed daily. For complete starvation, larvae were transferred to paper moistened with Ringers solution. For amino acid starvation, larvae were transferred to fly media lacking protein or amino acids. Overexpression of PI3K and Myc transgenes was performed using the heat-shock flp-out method [42]. Transgenes were induced by transferring larvae to a $37 \mathrm{C}$ room for $1.5 \mathrm{hrs}$ and then returning flies to $25 \mathrm{C}$ for the indicated postinduction recovery times. In each case controls were similarly heat-shocked larvae lacking the UAS transgene. Our microarray analysis indicated that this protocol led to a 20-30 fold induction of Myc mRNA and PI3K mRNA (Additional File 1). For analysis of TOR mutants, hatched larvae were transferred and raised in regular fly food for $48 \mathrm{~h}$ before being collected for RNA extraction. Control larvae were age-matched $\mathrm{w}^{1118}$ larvae.

\section{RNA extraction and microarray analysis}

For all microarray experiments, larvae were collected, snap-frozen and maintained at $-80 \mathrm{C}$ prior to RNA extraction. Total RNA was extracted with TRIzol reagent (Invitrogen) followed by RNeasy (Qiagen) clear up. cRNA targets were generated and coupled to either Cy3 or Cy5 fluorophores according to 'FHCRC Genomics Resource DNA Array Laboratory' protocols. Expression profiles were performed using spotted microarrays constructed from release 1 (6 K, Figs 1, 2, 3A, B, and 4 ) or 1 and 2 (12 K, Fig 3C, D) of the D. melanogaster Gene Collection (GEO GPL4285 = ' 6 k' and GEO GPL1908 = ' $12 \mathrm{k}$ '). Target label preparation and hybridization protocols are described elsewhere http://www. fhcrc.org/science/shared_resources/genomics/index.html. Hybridization and scanning were performed by the Fred Hutchinson Cancer Research Center Genomics Resource DNA Array Laboratory. Microarray images were quantified using GenePix Pro software (Axon Instruments).

\section{Statistics and Bioinformatics}

Data were generated from four or five independent replicates, with at least 2 replicates for each dye orientation. Spot intensities were filtered and removed if the values did not exceed 250 units above background or if a spot was flagged as questionable by the GenePix Pro software (Molecular Devices Corporation, Sunnyvale, CA). Spot-level ratios were $\log 2$ transformed and normalized using centralization method using Microsoft excel program to correct for observed intra-array intensity-dependent ratio biasing. Significance analysis of microarrays (SAM, http://www-stat.stanford.edu/ tibs/ SAM/) was used to select statistically significant data and a two-class paired test was conducted. A fold change threshold of 2-fold for starvation, PI3 kinase overexpression, TOR, and Myc mutants, and 1.7 foldthreshold for Myc overexpression, plus a false discovery rate (FDR) of $<5 \%$ were set as cut off to identify genes with statistically significant changes in expression. Heat maps were made using Cluster and TreeView program. http://rana.lbl.gov/EisenSoftware.htm.

Clusters of genes with similar expression patterns were generated using Genesis program http://genome. tugraz.at. Regulatory motifs were identified using MEME http://meme.sdsc.edu/meme4_1/cgi-bin/meme. cgi[43]. Upstream sequences of 500 bp plus 5'UTR of each gene from each cluster were used for motif search. GOMO (Gene Ontology for Motifs) [44] was used to analyze GO-terms significantly associated with genes containing motif1.

\section{GFP-reporter assays and primers}

The upstream regions of selected genes (cluster A CG768, CG1135, CG6311, CG32486; cluster B CG8470, CG6459, CG9539) were amplified by PCR from genomic DNA isolated from wildtype flies and subcloned into Stinger GFP transformation vector (obtained from Drosophila Genome Resource Center). The $5 \times$ motif1 reporter genes were constructed by cloning five tandem repeats of Motif1 into a Stinger GFP transformation vector [45]. GFP mRNA levels were measured by quantitative real-time RT-PCR using RNA extracted from whole larvae.

\footnotetext{
Additional file 1: List of genes affected in each microarray experiment. Gene names and associated GO terms are shown. Click here for file

[http://www.biomedcentral.com/content/supplementary/1471-2121-11-7S1.XLS ]
} 
Additional file 2: AA starvation induces a dynamic change in gene expression. All genes altered by AA starvation were clustered into one of 16 different groups based on their expression profiles. The plots represent fold change in expression level at different starvation/reefed time-points for each gene in the cluster. The pink line represents the average expression profile for each cluster. Green shade indicates different starvation time-points, red shade indicates re-feed timepoints. Click here for file

[ http://www.biomedcentral.com/content/supplementary/1471-2121-11-7S2.JPEG ]

Additional file 3: Examples of gene classes affected by AA starvation in Drosophila larvae. Heat maps depicting AA-starvation regulated genes involved lipid metabolism, carbohydrate metabolism, and TCA genes. Columns indicate expression changes at different AA starvation/re-feeding timepoints.

Click here for file

[http://www.biomedcentral.com/content/supplementary/1471-2121-11-7S3.JPEG ]

Additional file 4: Examples of gene classes affected by AA starvation in Drosophila larvae. Heat maps depicting AA-starvation regulated genes in proteolysis or autophagy. Columns indicate expression changes at different AA starvation/re-feeding timepoints. Click here for file

[ http://www.biomedcentral.com/content/supplementary/1471-2121-11-7S4.JPEG ]

Additional file 5: Examples of gene classes affected by AA starvation in Drosophila larvae. Heat maps depicting AA-starvation regulated genes involved in ribosome or protein synthesis. Columns indicate expression changes at different AA starvation/re-feeding timepoints.

Click here for file

[http://www.biomedcentral.com/content/supplementary/1471-2121-11-7S5.JPEG ]

Additional file 6: AA starvation has little effect on cell cycle genes. Graphs depicting fold changes in AA starvation-induced genes, AA starvation-repressed genes and cell cycle genes, in response to AA starvation and subsequent re-feeding. AA starvation has little effect in the expression of cell cycle genes.

Click here for file

[http://www.biomedcentral.com/content/supplementary/1471-2121-11-7S6.JPEG ]

Additional file 7: Comparison of different starvation protocols on gene expression in Drosophila larvae. Pie charts depicting overlap in both up-regulated (left) and down-regulated (right) gene expression following $24 \mathrm{~h}$ of a sucrose-only diet, complete starvation, or normal diet minus protein.

Click here for file

[http://www.biomedcentral.com/content/supplementary/1471-2121-11-7S7.JPEG ]

Additional file 8: GO-enrichment for genes affected by PI3 kinase overexpression.

Click here for file

[ http://www.biomedcentral.com/content/supplementary/1471-2121-11-7S8.PDF ]

Additional file 9: GOMO analysis of GO terms associated with motif 1 containing genes. The E-values and P-values are indicated for each GO term.

Click here for file

[http://www.biomedcentral.com/content/supplementary/1471-2121-11-7S9.PDF ]

\section{Acknowledgements}

We thank Jim McGhee, Jeb Gaudet and William Brook for comments on the manuscript. We thank Tom Neufeld for the TOR mutant line. This work was supported by NIH grant GM51186 to B.A.E and a CIHR grant to S.S.G. S.S.G is a Scholar of the Alberta Heritage Foundation for Medical Research and an Alberta Cancer Board New Investigator.

\section{Author details}

${ }^{1}$ Fred Hutchinson Cancer Research Center, Seattle, WA 98109, U.S.A. ${ }^{2} \mathrm{Clark}$ H. Smith Brain Tumour Center, Southern Alberta Cancer Research Institute, Calgary, Alberta, T2N 4N1, Canada. ${ }^{3}$ Department of Biochemistry and Molecular Biology, University of Calgary, Calgary, Alberta, T2N 4N1, Canada.

\section{Authors' contributions}

$B A E$ and $L L$ conceived of the project. BAE, LL and SSG designed the experiments. $L L$ performed the microarray analyses and helped edit the manuscript. SSG performed the reporter assays in Fig 5, and drafted the manuscript. LL and SSG analyzed the data. All authors read and approved the final manuscript.

Received: 9 October 2009

Accepted: 20 January 2010 Published: 20 January 2010

\section{References}

1. Lopez-Maury L, Marguerat S, Bahler J: Tuning gene expression to changing environments: from rapid responses to evolutionary adaptation. Nat Rev Genet 2008, 9(8):583-593.

2. Zaman S, Lippman SI, Zhao X, Broach JR: How Saccharomyces Responds to Nutrients. Annual Review of Genetics 2008, 42(1):27-81

3. Jorgensen P, Nishikawa JL, Breitkreutz BJ, Tyers M: Systematic identification of pathways that couple cell growth and division in yeast. Science 2002, 297(5580):395-400

4. Jorgensen P, Rupes I, Sharom JR, Schneper L, Broach JR, Tyers M: A dynamic transcriptional network communicates growth potential to ribosome synthesis and critical cell size. Genes Dev 2004, 18(20):2491-2505.

5. Britton JS, Edgar BA: Environmental control of the cell cycle in Drosophila: nutrition activates mitotic and endoreplicative cells by distinct mechanisms. Development (Cambridge, England) 1998, 125(11):2149-2158

6. Britton JS, Lockwood WK, Li L, Cohen SM, Edgar BA: Drosophila's insulin/ PI3-kinase pathway coordinates cellular metabolism with nutritional conditions. Dev Cell 2002, 2(2):239-249.

7. Oldham S, Hafen E: Insulin/IGF and target of rapamycin signaling: a TOR de force in growth control. Trends Cell Biol 2003, 13(2):79-85.

8. Wullschleger $\mathrm{S}$, Loewith $\mathrm{R}$, Hall MN: TOR signaling in growth and metabolism. Cell 2006, 124(3):471-484

9. De Virgilio C, Loewith R: The TOR signalling network from yeast to man. Int J Biochem Cell Biol 2006, 38(9):1476-1481.

10. Saucedo $\amalg$, Gao X, Chiarelli DA, Li L, Pan D, Edgar BA: Rheb promotes cell growth as a component of the insulin/TOR signalling network. Nat Cell Biol 2003, 5(6):566-571.

11. Stocker $H$, Radimerski $T$, Schindelholz B, Wittwer F, Belawat $P$, Daram $P$, Breuer S, Thomas G, Hafen E: Rheb is an essential regulator of S6K in controlling cell growth in Drosophila. Nat Cell Biol 2003, 5(6):559-565.

12. Shaw RJ, Cantley LC: Ras, $\mathrm{PI}(3) \mathrm{K}$ and mTOR signalling controls tumour cell growth. Nature 2006, 441(7092):424-430.

13. Grewal SS: Insulin/TOR signaling in growth and homeostasis: $A$ view from the fly world. Int J Biochem Cell Biol 2008, 41(5):1006-1010.

14. Zinke I, Schutz CS, Katzenberger JD, Bauer M, Pankratz MJ: Nutrient control of gene expression in Drosophila: microarray analysis of starvation and sugar-dependent response. Embo J 2002, 21(22):6162-6173.

15. Buch S, Melcher C, Bauer M, Katzenberger J, Pankratz MJ: Opposing effects of dietary protein and sugar regulate a transcriptional target of Drosophila insulin-like peptide signaling. Cell Metab 2008, 7(4):321-332.

16. Teleman AA, Hietakangas V, Sayadian AC, Cohen SM: Nutritional control of protein biosynthetic capacity by insulin via Myc in Drosophila. Cell Metab 2008, 7(1):21-32.

17. Gershman B, Puig O, Hang L, Peitzsch RM, Tatar M, Garofalo RS: Highresolution dynamics of the transcriptional response to nutrition in Drosophila: a key role for dFOXO. Physiol Genomics 2007, 29(1):24-34

18. Grewal SS, Evans JR, Edgar BA: Drosophila TIF-IA is required for ribosome synthesis and cell growth and is regulated by the TOR pathway. J Cell Biol 2007, 179(6):1105-1113. 
19. Wang B, Goode J, Best J, Meltzer J, Schilman PE, Chen J, Garza D, Thomas JB, Montminy M: The insulin-regulated CREB coactivator TORC promotes stress resistance in Drosophila. Cell Metab 2008, 7(5):434-444.

20. Zhang H, Stallock JP, Ng JC, Reinhard C, Neufeld TP: Regulation of cellular growth by the Drosophila target of rapamycin dTOR. Genes Dev 2000, 14(21):2712-2724.

21. Grewal SS, Li L, Orian A, Eisenman RN, Edgar BA: Myc-dependent regulation of ribosomal RNA synthesis during Drosophila development. Nat Cell Biol 2005, 7(3):295-302.

22. Pierce SB, Yost C, Anderson SA, Flynn EM, Delrow J, Eisenman RN: Drosophila growth and development in the absence of dMyc and dMnt. Dev Biol 2008, 315(2):303-316.

23. Ohler U, Liao GC, Niemann H, Rubin GM: Computational analysis of core promoters in the Drosophila genome. Genome Biology 2002, 3: research0087.1-0087.12.

24. Tettweiler G, Miron M, Jenkins M, Sonenberg N, Lasko PF: Starvation and oxidative stress resistance in Drosophila are mediated through the elF4E-binding protein, d4E-BP. Genes Dev 2005, 19(16):1840-1843.

25. Teleman AA, Chen YW, Cohen SM: 4E-BP functions as a metabolic brake used under stress conditions but not during normal growth. Genes Dev 2005, 19(16):1844-1848.

26. Teleman AA, Maitra S, Cohen SM: Drosophila lacking microRNA miR-278 are defective in energy homeostasis. Genes Dev 2006, 20(4):417-422.

27. Scott RC, Schuldiner O, Neufeld TP: Role and regulation of starvationinduced autophagy in the Drosophila fat body. Dev Cell 2004, 7(2):167-178.

28. Scott RC, Juhasz G, Neufeld TP: Direct induction of autophagy by Atg1 inhibits cell growth and induces apoptotic cell death. Curr Biol 2007, 17(1):1-11.

29. Edgar BA, Orr-Weaver TL: Endoreplication cell cycles: more for less. Cell 2001, 105(3):297-306.

30. Polymenis M, Schmidt EV: Coupling of cell division to cell growth by translational control of the G1 cyclin CLN3 in yeast. Genes Dev 1997, 11(19):2522-2531.

31. Guertin DA, Guntur KV, Bell GW, Thoreen CC, Sabatini DM: Functional genomics identifies TOR-regulated genes that control growth and division. Curr Biol 2006, 16(10):958-970.

32. Hall DJ, Grewal SS, de la Cruz AF, Edgar BA: Rheb-TOR signaling promotes protein synthesis, but not glucose or amino acid import, in Drosophila. BMC Biol 2007, 5(1):10.

33. Hennig KM, Colombani J, Neufeld TP: TOR coordinates bulk and targeted endocytosis in the Drosophila melanogaster fat body to regulate cell growth. J Cell Biol 2006, 173(6):963-974.

34. Burgering BM: A brief introduction to FOXOlogy. Oncogene 2008, 27(16):2258-2262.

35. Junger MA, Rintelen F, Stocker H, Wasserman JD, Vegh M, Radimerski T, Greenberg ME, Hafen E: The Drosophila forkhead transcription factor FOXO mediates the reduction in cell number associated with reduced insulin signaling. J Biol 2003, 2(3):20.

36. Demontis F, Perrimon N: Integration of Insulin receptor/Foxo signaling and dMyc activity during muscle growth regulates body size in Drosophila. Development (Cambridge, England) 2009, 136(6):983-993.

37. Saucedo $\sqcup$, Edgar BA: Why size matters: altering cell size. Curr Opin Genet Dev 2002, 12(5):565-571.

38. DeBerardinis RJ, Lum JJ, Hatzivassiliou G, Thompson CB: The biology of cancer: metabolic reprogramming fuels cell growth and proliferation. Cell Metab 2008, 7(1):11-20.

39. Ruggero D, Montanaro L, Ma L, Xu W, Londei P, Cordon-Cardo C, Pandolfi PP: The translation factor elF-4E promotes tumor formation and cooperates with c-Myc in lymphomagenesis. Nat Med 2004, 10(5):484-486.

40. Deato MD, Tjian R: Switching of the core transcription machinery during myogenesis. Genes Dev 2007, 21(17):2137-2149.

41. Orian A, van Steensel B, Delrow J, Bussemaker HJ, Li L, Sawado T, Williams E, Loo LW, Cowley SM, Yost C, et al: Genomic binding by the Drosophila Myc, Max, Mad/Mnt transcription factor network. Genes Dev 2003, 17(9):1101-1114.

42. Elliott DA, Brand AH: The GAL4 system: a versatile system for the expression of genes. Methods Mol Biol 2008, 420:79-95.

43. Bailey TL, Elkan C: Fitting a mixture model by expectation maximization to discover motifs in biopolymers. Proc Int Conf Intell Syst Mol Biol 1994, 2:28-36.
44. Boden M, Bailey TL: Associating transcription factor-binding site motifs with target GO terms and target genes. Nucleic Acids Res 2008, 36(12):4108-4117.

45. Barolo S, Castro B, Posakony JW: New Drosophila transgenic reporters: insulated P-element vectors expressing fast-maturing RFP. Biotechniques 2004, 36(3):436-440.

doi:10.1186/1471-2121-11-7

Cite this article as: Li et al:: Nutritional control of gene expression in Drosophila larvae via TOR, Myc and a novel cis-regulatory element. BMC Cell Biology 2010 11:7.

\section{Submit your next manuscript to BioMed Central and take full advantage of:}

- Convenient online submission

- Thorough peer review

- No space constraints or color figure charges

- Immediate publication on acceptance

- Inclusion in PubMed, CAS, Scopus and Google Scholar

- Research which is freely available for redistribution 